\title{
Un mundo para la gente: estrategias de diseño para la innovación
}

Alejandro Rivera Plata*

\section{Resumen}

En este documento se describen los pros y los contras de los conceptos destrucción creativa y discontinuidad. Estos hacen parte del cuerpo teórico de la innovación y son considerados como elementos sustanciales para comprender la innovación en su dimensión dinámica y social. En el análisis crítico de estos términos surgen los conceptos destrucción destructiva y fragmentación social, hecho que evidencia algunas de las problemáticas que conllevan los procesos de innovación en la dimensión social. Del entendimiento de estos fenómenos deriva la necesidad de concebir el uso de procesos de innovación, cocreación o innovación abierta entre ellos, en calidad de mecanismos y dinámicas que respetan los intereses particulares y propenden por la integración de las organizaciones y los individuos que las constituyen con las dinámicas del mercado de una manera armónica. De esta manera, el diseño y el diseñar emergen como instrumentos para la integración, el desarrollo de la innovación social y como alternativas de innovación para la gente, mas no para el mercado.

Palabras clave: acceso a la innovación, destrucción creativa, destrucción destructiva, innovación

\section{Abstract}

This document describes the pros and cons of the concepts of creative destruction and discontinuity. These are part of the theoretical body of innovation and are considered as substantial elements to understand innovation in its dynamic and social dimension. In the critical analysis of these terms, the concepts destructive destruction and social fragmentation arise, a fact that evidences some of the problems involved in innovation processes in the social dimension. From the understanding of these phenomena derives the need to conceive the use of innovation, co-creation or open innovation processes among them, as mechanisms and dynamics that respect the particular interests and tend to the integration of organizations and individuals that constitute them with market dynamics in a harmonious way. In this way, design and design emerge as instruments for integration, the development of social innovation and as alternatives for innovation for people, but not for the market.

Keywords: access to innovation, creative destruction, destructive destruction, innovation

\footnotetext{
* Diseñador Gráfico y Especialista en Pedagogía del Diseño de la Universidad Nacional de Colombia. Docente en la Corporación Nacional Unificada de Educación Superior (cuN). Contacto: danilo_rivera@cun.edu.co
} 


\section{Introducción}

Las organizaciones empresariales establecen relaciones entre ellas, el mercado y sus consumidores; esto determina continuamente retos para su propia supervivencia. Además, factores como la globalización complejizan su función y las ubican en una situación en la que la competitividad se convierte en el objetivo a seguir. En este estado de las cosas, la innovación adquiere una relevancia vital para las organizaciones $y$, en este sentido, es asumida sin ningún miramiento. Respecto a esta conducta, se pueden apreciar circunstancias en las que la innovación, sin restricciones, se convierte en un factor que afecta a los individuos y otras organizaciones sociales que se encuentran relacionadas con estas organizaciones comerciales, al plantear la duda sobre las circunstancias que rodean a la innovación cuando esta se torna negativa y si es posible conciliar la innovación, entendida en su carácter comercial, con los intereses de los grupos sociales que resultan afectados, de manera que se pueda hablar de un criterio para la concepción de innovaciones.

El objetivo del presente documento es, en primera instancia, reconocer las motivaciones que conducen a la necesidad de innovar, para luego establecer las circunstancias en las que la innovación se puede tornar negativa debido a las consecuencias de su acción. Por último, pretende apostar por las posibles soluciones a esta situación que, hoy en día, están siendo abordadas desde una perspectiva social.

\section{La invitación a innovar}

Desde la caída del bloque soviético en los años noventa, el capitalismo se erigió, sin lugar a duda, como el sistema económico dominante y en el que se hallan inmersas prácticamente todas las sociedades humanas actuales. De esta manera, las dinámicas y tensiones propias del capitalismo terminan siendo parte de la cotidianidad de millones de seres humanos, por lo que entenderlas se convierte en un asunto de supervivencia.

Se destaca en esta cotidianidad vívida del capitalismo la noción de empresa. Entendida como unidad de producción, toda empresa se encuentra determinada por su capacidad de ofertar al mercado su producción y garantizar su propia subsistencia mediante el intercambio de sus productos. Este proceso de sostenimiento, llamémoslo así, exige que las empresas estén cons- tantemente en búsqueda de ventajas frente a las otras ofertas que se encuentran en el mercado.

Respecto a la constante búsqueda de ventajas competitivas, el economista Joseph Schumpeter (1944) señaló que las empresas que se van volviendo obsoletas son remplazadas por aquellas que innovan porque estas últimas presentan un beneficio más evidente para productores y consumidores, y, por ende, son las más deseables para la economía. Este proceso de remplazo es denominado por el autor como destrucción creativa. Según él, la destrucción creativa activa el comercio al promover el desarrollo de nuevas mercancías, nuevas formas de producción, nuevos modelos de negocio, etc. Esta activación se convierte en una acción deseable para el mercado, ya que se puede ver reflejada en progreso económico. 
En este mismo sentido, Peter Drucker (1969) afirma que el principio de discontinuidad ${ }^{1}$ en el mercado es el que se encarga de administrar y controlar la destrucción creativa. En otras palabras, se trata del principio que regula y estimula la aparición de nuevas empresas, así como la salida de aquellas menos productivas.

Así, las dinámicas impuestas por las mismas relaciones entre las organizaciones en el mercado proponen unos ciclos de continua reconfiguración de ellas y del mercado mismo. Estos procesos, conocidos como ciclos schumpeterianos, exigen que las organizaciones desarrollen estrategias que les permitan generar continuamente ventajas competitivas. Asimismo, estos ciclos y discontinuidades son dinamizadores del desarrollo empresarial y social (Franch, 1996), motivo por el que son vistos como deseables y son incentivados desde las mismas prácticas empresariales y las políticas públicas gubernamentales.

\section{Los que no}

Los procesos de desarrollo impuestos por las dinámicas del mercado responden a los principios de su funcionamiento interno; sin embargo, estos principios difieren de los de las organizaciones: mientras los mercados incentivan un continuo relevo de productos en busca de una ventaja sobre la competencia (Leal y Quero, 2011), las organizaciones, según Max Weber (1964), tienden al encuentro y consolidación de un medio estable para su desarrollo. Si bien, el cambio está presente, en ocasiones su ritmo resulta imperceptible para algunos de quienes lo viven.

Desde la perspectiva de la empresa concebida como una organización, los cambios o discontinuidades impuestos por el mercado, como ya se ha expuesto, se traducen en la necesidad de ejecutar cambios tanto en la oferta de productos como dentro de las organizaciones productoras. Al erigirse como un factor inherente a las organizaciones contemporáneas, se podría suponer que el cambio es adoptado de manera natural por los miembros de las organizaciones; sin embargo, este también suele generar conflictos con los individuos. Por ejemplo, según Salvador García (1997), los cambios que se asocian a la implementación de herramientas como la calidad total, la reingeniería y los sistemas administrativos o de información, entre otros, usualmente están condenados al rechazo y al fracaso. ¿Por qué? En buena medida, porque muchas veces son profundamente incomprendidos y por las confusiones que generan. Es de señalar que los cambios impactan fuertemente en las creencias y los valores culturales preexistentes en las organizaciones, hecho que, a su vez, resuena en las rupturas e incertidumbres percibidas y vividas por los individuos que pertenecen a ellas. De esta manera, los cambios y, entre ellos, las innovaciones, también pueden convertirse en detonantes de conflictos entre los miembros de una organización o, incluso, en el fuero interno de los mismos individuos.

Un ejemplo de los conflictos detonados por los cambios entre los individuos de una organización es la implementación de las tecnologías de la información. Existen varios estudios que 
evidencian la fragmentación social derivada del acceso a las innovaciones en estas tecnologías, pues este suele separar a quienes acceden a ellas de aquellos a los que les son negadas. Núñez recuerda lo dicho por el sociólogo Manuel Castells en el marco del encuentro del Grupo de los Siete en Bruselas:

la difusión y desarrollo de ese sistema tecnológico ha cambiado la base material de nuestras vidas, por tanto la vida misma, en todos sus aspectos: en cómo producimos, cómo y en qué trabajamos, cómo y qué consumimos, cómo nos educamos, cómo nos informamos-entretenemos, cómo vendemos, cómo nos arruinamos, cómo gobernamos, cómo hacemos la guerra y la paz, cómo nacemos y cómo morimos, y quién manda, quién se enriquece, quién explota, quién sufre y quién se margina. Las nuevas tecnologías de información no

\section{El problema es personal}

La concepción de la producción para todos constituye una parte clave de lo que define el mundo contemporáneo. Desde esta perspectiva, la integración de todo el mundo en una actitud receptiva hacia la producción misma, sus modelos y procesos, y la innovación se convierte en una de sus máximas aspiraciones. Aun así, existen obstáculos que se pueden reconocer en el fracaso de la implementación de estrategias de innovación en las organizaciones.

En los estudios sobre innovación educativa de Rimari (s. f.) y Ojeda y Perales (2011), y los de implementación de innovaciones en empresas de Guerra, en México y en Chile, es posible determinan lo que pasa en la sociedad, pero cambian tan profundamente las reglas del juego que debemos aprender de nuevo, colectivamente, cuál es nuestra nueva realidad, o sufriremos, individualmente, el control de los pocos (países o personas) que conozcan los códigos de acceso a las fuentes de saber y poder. $(2013$, p. 56)

En este mismo sentido, Umberto Eco, en su libro Apocalípticos e integrados (1995), plantea una división entre aquellos que han adoptado la cultura que circula entorno a las tecnologías actuales de comunicación e incentiva la producción y el consumo de sus propios productos, y aquellos que las rechazan y reniegan de su legitimidad desde su posición de defensa de las tradiciones. Incluso va más allá de esta fractura al revelar que esta división, más allá de su contenido, suele validarse simplemente por la oposición al otro.

distinguir que las barreras que desaceleran la innovación responden a factores internos y externos de las organizaciones. Según de la Torre (1987), los factores más complejos que detienen la innovación corresponden a las actitudes de los individuos y su miedo al cambio, siendo estos miedos expresiones de una suerte de autodefensa ante la posibilidad de exponer sus emociones o necesidades instintivas de su yo profundo. Esto genera una lucha con sus impulsos, esta confrontación en busca de controlar la "realidad", según el mismo de la Torre, termina por agotar al individuo, causándole fatiga, ansiedad y expresión, estados que finalmente se interponen a cualquier conducta de cambio. 
Entonces, se pueden distinguir dos situaciones que le impiden al individuo aceptar el cambio y la integración de la innovación:

1. El conocimiento previo: la innovación es un proceso acumulativo de conocimiento, de manera que los productos de la innovación están, en su mayoría, fundamentados en otros saberes y formas de hacer que son necesarios para su implementación. La ausencia o debilidad de estos últimos hace difícil su incorporación y, en consecuencia, emerge el rechazo a la innovación.

2. El esfuerzo de alfabetización: la complejidad de una innovación puede requerir la realización de un esfuerzo grande para su compresión e implementación; sin embargo, no todos los individuos están dispuestos a realizar dichos esfuerzos. Factores como el tiempo pueden resultar determinantes para decidir no adoptar una innovación y, por ello, descartar cualquier intención de acoger una.

En ambos casos, las circunstancias que rodean a los individuos y sus propias particularidades resultan importantes para determinar la posibilidad de incorporar o no una innovación en una organización, incluso más allá de los beneficios que la propia innovación representa para la comunidad.

\section{Las paradojas de la innovación}

Si se pasan por alto los posibles inconvenientes a los que se puede enfrentar una innovación en su proceso de implementación o distribución, los beneficios de la destrucción creativa y las discontinuidades parecen inermes; sin embargo, la misma lógica de estos procesos plantea algunas paradojas.

Como sabemos, los ciclos de desarrollo de las innovaciones buscan continuamente la renovación de la oferta en el mercado o la apertura de nuevos mercados. La continua búsqueda de beneficios en este modelo propone una competencia que día a día se va haciendo más reñida. Respecto a esta dinámica de competitividad en la producción de la modernidad, Marshall Berman (1988) explica que la producción moderna está caracterizada por la celeridad y el consumo moderno está caracterizado por el vacío. Con esto en mente, la situación es la siguiente: en la modernidad, el público consume rápidamente lo que le es ofrecido como innovación, hecho que, en consecuencia, induce a la industria a producir aún más rápido estas innovaciones para mantener el consumo y obtener una posición de ventaja en la oferta, de manera que se acelera aún más el consumo. Esta situación describe un círculo vicioso que define el vacío, descrito por Berman, del consumo sin sentido; un consumo carente de un significado más allá del consumo mismo. De la misma manera, este círculo vicioso cuestiona la producción y la innovación en el sentido de debatir la relevancia del acto innovador.

Un ejemplo de carencia de trasfondo en la producción es el uso del precepto de la obsolescencia programada en el desarrollo de producto. Mayra Ruiz y Zylath Romero reconocen dos tipos de obsolescencia programada:

Obsolescencia programada objetiva o funcional. Se basa en la vida útil o duración real del producto o mercancía, que 
ha sido previamente estimada. El usuario está obligado a comprar un nuevo producto, ya que el que posee no le sirve.

Obsolescencia programada subjetiva o no funcional. Se basa en los trabajos de marketing; el producto sigue siendo útil pero el propietario quiere renovarlo por uno más reciente o atractivo, lo que para él significa más comodidad, confort y solvencia ante la sociedad. (2014, p. 133)

En el primer caso, el usuario se ve obligado a descartar su vinculación con el objeto. En el segundo, el usuario es seducido a descartar su vínculo con el objeto. En ambos casos, la supresión del vínculo implica una barrera para la consideración de un significado transcendente de estos objetos y de los que vendrán a remplazarlos, que tendrán el mismo final.

También es posible analizr los casos de los afectados por los procesos de destrucción creativa. Schumpeter argumentó que siempre habrán afectados por los procesos de destrucción creativa; incluso, sus ciclos establecen la existencia de profundas crisis de carácter periódico. Desde la perspectiva más racional, estas crisis y afectados son apenas lógicos y necesarios para lograr el desarrollo propuesto por el modelo capitalista. Pero, sobre este aspecto cabe también plantear la duda referente a los beneficios de la innovación: ¿es posible que la destrucción creativa destruya más de lo que crea?

El sociólogo portugués Boaventura de Sousa Santos se cuestiona sobre las bondades de la destrucción creativa en algunos campos del desarrollo humano, especialmente en el desarrollo del conocimiento científico. Al respecto, declara:
La ciencia moderna asumió desde su inicio una postura que Schumpeter habría de atribuir más tarde al capitalismo: la capacidad de destrucción creadora. En términos epistemológicos, tal postura consiste en la propia idea de revolución científica como una quiebra radical con todos los conocimientos anteriores [...]. Al rechazar todos los conocimientos alternativos, la ciencia moderna se reveló como una productora de basura, condición que todos nosotros [...] de igual modo compartimos. Esta es una dimensión más de la referida epistemología de la basura, y también de una economía política simbólica de producción de residuos en la ciencia moderna.

Dos interrogaciones se imponen a este respecto: ¿Cuánto desperdicio es preciso hacer para producir consecuencias científicas? ¿Quién sufre más con la polución de ahí resultante? (2003, p. 273)

Desde esta perspectiva, Santos (2003) define la noción de destrucción destructiva como el vendaval de la destrucción creativa que interviene de manera indiscriminada en la cultura y las sociedades con lo que se destruye y encubre todo a su alrededor. El portugués construye esta definición haciendo uso de la analogía que supondría el uso de excavadoras mecánicas en la exploración arqueológica. Al respecto, Ignacio Ayestarán establece que

el dilema de la excavadora ha tenido un tratamiento específico en arqueología, a fin de evitar un conocimiento que destruye los vestigios y que solo contribuye a la epistemología del desperdicio. 
Sin embargo, en economía, parece haberse obviado esta dificultad [...]. Por eso mismo, y contrariamente a lo que acontece en la arqueología, en economía no fue concebida estrategia alternativa alguna. La ceguera frente a este dilema aumenta la posibilidad de que la destrucción creadora de la economía convencional pase a

\section{La mesa para todos}

Al reconocer, entonces, que toda innovación trae consigo un acto de reconfiguración de las condiciones que la rodean, acto definido como una destrucción, y tras considerar que dicha destrucción puede convertirse en un acto negativo para varios de los actores que la circundan -entre ellos, los grupos sociales que se pretende que adopten dichas innovaciones, las comunidades de trabajo que se verán en desventaja y los mercados en los que se implantan dichas innovaciones-, se hace relevante la necesidad de crear mecanismos que propendan por la integración de las innovaciones de manera que se pueda garantizar el acceso a la innovación a las comunidades afectadas y la armonización de estas con los intereses particulares de los individuos que las integrarán a su cultura. En este mismo sentido, es preciso que sobre estos mecanismos de acceso a la innovación se plantee la necesidad de integrar aspectos como el conocimiento ancestral y el patrimonio cultural de las comunidades afectadas, a fin de garantizar su protección y proyección a futuro.

Núñez retoma el planteamiento de Manuel Castells sobre esta circunstancia, que implica buscar caminos para adoptar la innovación y el proceso de destrucción creativa: "no podemos desarrollar su dimensión creativa y escapar a sus efectos potencialmente devastadores sin afrontar ser, tan sólo, destrucción destructiva.

(2011, p. 70)

Estas circunstancias plantean varias dudas respecto al hecho de asumir acríticamente la innovación en calidad de sinónimo de desarrollo y beneficios. Comprender estas paradojas planteadas conlleva dar una orientación adecuada a la actitud innovadora de tal manera que asegure más beneficios que perjuicios.

colectivamente quiénes somos y qué queremos. Lo que tal vez [...] debiera plantearse es cómo reequilibrar nuestro superdesarrollo tecnológico y nuestro subdesarrollo social" (2013, pp. 57-58).

En esta instancia, la búsqueda de dicho equilibrio estaría medida por el reconocimiento de los individuos y su cultura como parte fundamental de los procesos de desarrollo de las innovaciones. Como lo define Santos,

el conflicto entre la globalización neoliberal y la globalización contrahegemónica anticapitalista representa un campo social relativamente poco cartografiado que se caracteriza por tener riesgos de opresión, sufrimiento humano y destrucción, difíciles o hasta imposibles de sortear, así como por tener posibilidades y oportunidades nuevas y no imaginadas para una política emancipadora. (2010, p. 80)

A su vez, entender al individuo como parte de las organizaciones implica el diseño de estrategias que promuevan el desarrollo de las organizaciones y de sus capacidades de innovar. Cruz cita a Alfred Sloan para explicar que 
el desarrollo organizacional se hace indispensable en nuestras instituciones sociales contemporáneas y del futuro, por la lucha que tienen que llevar a cabo para sobrevivir en las condiciones del medio que corresponden a un cambio crónico en las instituciones sociales que coordinan los asuntos de casi todas las organizaciones humanas. (1999, párr. 15)

En este mismo sentido, también se hace necesaria la comprensión, esta vez, por parte de las mismas comunidades afectadas, de las dinámicas del mundo actual en las que el cambio se convierte en una constante, en un inevitable devenir de las cosas. Al respecto, Pedro Schwartz (30 de mayo del 2003) destaca que las estructuras innovadoras pueden surgir sin necesariamente destruir la capacidad de ganarse la vida de los acostumbrados al modo antiguo de hacer las cosas, si ellos muestran adaptabilidad al cambio.

Otros aspectos relevantes para el desarrollo de estas integraciones son los dados por los procesos de ideación y de implementación de las innovaciones. Como lo exponen Sanders y Stappers (2008), los procesos de diseño participativo y de diseño centrado en el usuario han resultado útiles no solo por la creación de productos y servicios en sí, sino por la generación de información que ha permitido comprender al usuario y el entorno de uso de los objetos más profundamente y de manera tal que los productos de estos procesos resultan ser más pertinentes para el usuario y tienden a poseer un valor mayor que otros, ya que se perciben como frutos de su propia cultura (saberes y formas de hacer), a la vez que desarrollan habilidades en los equipos de diseño encaminadas a comprender al usuario en su cotidianidad.

De la misma forma, el hecho de hacer partícipes a los individuos en los procesos de creación hace que el producto mismo del proceso adquiera valor antes de ser publicado, lo que, a su vez, dispone en doble vía los procesos de integración: de los objetos a los individuos y de los individuos a los objetos.

\section{Un mundo para la gente}

La idea de formas de desarrollo que se ubiquen en el contexto de la cotidianidad de las personas que pretenden impactar implica pensarse el diseño desde otras ópticas, cuyo centro de interés es el empoderamiento de las comunidades. Desde esta perspectiva, el diseño y el diseñar se convierten en estrategias para materializar unas formas del diseño que emergen de los mismos conocimientos y habilidades de las comunidades que se pretende beneficiar. En este sentido, Víctor Papanek define el diseño como "un atributo humano básico para ayudar a la autorrealización autónoma" (2005, p. 306). Esta noción convierte

al diseñador en agente del diseño, en la medida en que él es el actor encargado de detonar al diseño mismo, pero no es condición para su materialización.

El diseño sin diseñadores, o mejor, el diseño en el que intervienen como diseñadores las propias comunidades, es una óptica que impregna a la actividad del diseño de un objetivo con mayor alcance que el diseño enfocado en productos. Se trata de un tipo de diseño que se entiende como actor en la trama social y que, en esta condición, posee una potencia de acción en la cotidianidad 
de las personas, en la medida en que se presta para la configuración de los entornos artificiales de actividad de las sociedades (Rapoport, 1982).

Amparados en este horizonte, se puede predecir una transformación de los entornos con mayor sentido que las implantaciones de diseño tradicionales, en las que se pretenden instalar las soluciones de diseño que han sido estructuradas desde visiones deslocalizadas y con una carga de precedentes (cultura) distinta a la de la situación que se busca intervenir. El diseño localizado posee sentido por su misma condición localizada; el diseño autogestionado posee un valor cultural que es definido por los mismos preceptos y valores de quienes diseñan, pues responde a sus propias inquietudes y lo hace desde sus mismas ópticas. Es un diseño que está pensado por y para la gente; para darle forma a un mundo para la gente.

\section{Referencias}

Ayestarán, I. (2011). Epistemología de la innovación social y de la destrucción creativa. Utopía y Praxis Latinoamericana, 16(54), 67-91.

Berman, M. (1988). Todo lo sólido se desvanece en el aire. Buenos Aires: Siglo XXI.

Cruz, A. de la. (1999). Problemas y principios para el desarrollo de las organizaciones modernas. Gestión en el Tercer Milenio, 2(3). Recuperado de https:/ / bit.ly/2ZFH1OH

Drucker, P. (1969). The Age of Discontinuity. New York: Harper \& Row.

Eco, U. (1995). Apocalípticos e integrados. Barcelona: Tusquets.

Franch, J. (1996). Economía a vuelapluma. Madrid: Eilea.

García, S. (1997). La dirección por valores. Madrid: McGraw Hill.

Leal, A. y Quero, M. (2011). Manual de marketing y comunicación cultural. Cadiz: Universidad de Cadiz. Recuperado de https:/ / bit.ly/2vgu9o5

Núñez, C. (2013). Pub-licidad: Simbología de masas. Madrid: Planeta.

Ojeda, F. y Perales, F. (2011). Buenas prácticas en el uso de las Tic en la enseñanza de la Biología y la Geología. En P. Cañal (coord.), Biología y geología. Investigación, innovación y buenas prácticas. Vol. III (pp. 121-144). Barcelona: Graó. 
Papanek, V. (2005). Edugrafologías. En Michael Bierut (ed.), Fundamentos del diseño (pp. 302-307). Buenos Aires: Infinito.

Rapoport, A. (1982). The Meaning of the Built Environment: A Nonverbal Communication Approach. Phoenix: University of Arizona Press.

Rimari, W. (s. f.). La innovación educativa. Un instrumento de desarrollo [recurso en línea]. Recuperado de https:// bit.ly/39odMV8

Ruiz, M. y Romero, Z. (2014). La responsabilidad social empresarial y la obsolescencia programada. Revista Ciencias Estratégicas, 6(1), 127-138.

Sanders, E. y Stappers, P. (2008). Co-creation and the new landscapes of design. Co-design, 4(1), $5-18$.

Santos, B. (2003). Crítica de la razón indolente: contra el desperdicio de la experiencia. Volumen I. Para un nuevo sentido común: la ciencia, el derecho y la política en la transición paradigmática. Bilbao: Desclée de Brouwer.

Santos, B. (2010). Descolonizar el saber, reinventar el poder. Montevideo: Trilce.

Schumpeter, J. (1944). Ensayos sobre el ciclo económico. México D. F.: Fondo de Cultura Económica.

Schwartz, P. (30 de mayo del 2003). Destrucción creadora. Libertad Digital, recuperado de https:// bit.ly/2Sc2ZaV

Torre, S. de la. (1987). Educar en la creatividad. Madrid: Marcea.

Weber, M. (1964). Economía y sociedad. Esbozo de sociología comprensiva. México D. F.: Fondo de Cultura Económica. 